\title{
General-relativistic rotation laws in rotating fluid bodies
}

\author{
Patryk Mach and Edward Malec \\ Instytut Fizyki im. Mariana Smoluchowskiego, Uniwersytet Jagielloński, Eojasiewicza 11, 30-348 Kraków, Poland
}

\begin{abstract}
We formulate new general-relativistic extensions of Newtonian rotation laws for self-gravitating stationary fluids. They have been used to re-derive, in the first post-Newtonian approximation, the well known geometric dragging of frames. We derive two other general-relativistic weak-field effects within rotating tori: the recently discovered dynamic anti-dragging and a new effect that measures the deviation from the Keplerian motion and/or the contribution of the fluids selfgravity. One can use the rotation laws to study the uniqueness and the convergence of the post-Newtonian approximations, and the existence of the post-Newtonian limits.
\end{abstract}

PACS numbers: 04.20.-q, 04.25.Nx, 04.40.Nr, 95.30.Sf

\section{INTRODUCTION}

Stationary Newtonian hydrodynamic configurations are characterized by a variety of rotation curves. The angular momentum per unit mass $j$ can be any function of $r$, where $r$ is the distance from the rotation axis. Other restrictions arise from stability considerations [1]. In contrast to that, for a long time the only known rotation law in general-relativistic hydrodynamics had been that with $j$ as a linear function of the angular velocity. Recently Galeazzi, Yoshida and Eriguchi [2] have found a nonlinear angular velocity profile, that may approximate the Newtonian monomial rotation curves $\Omega_{0}=w / r^{\lambda}$ in the nonrelativistic limit. In this paper we define general-relativistic rotation curves $j=j(\Omega)$ that in the nonrelativistic limit exactly coincide with $\Omega_{0}=w / r^{\lambda}$ $(0 \leq \lambda \leq 2, \lambda \neq 1)$. We are able to obtain the generalrelativistic Keplerian rotation law that possesses the first post-Newtonian limit (1PN) and exactly encompasses the solution corresponding to the massless disk of dust in the Schwarzschild spacetime.

\section{HYDRODYNAMICAL EQUATIONS}

We recapitulate, following [3] , the equations of generalrelativistic hydrodynamics. Einstein equations, with the signature of the metric $(-,+,+,+)$, read

$$
R_{\mu \nu}-g_{\mu \nu} \frac{R}{2}=8 \pi \frac{G}{c^{4}} T_{\mu \nu}
$$

where $T_{\mu \nu}$ is the stress-momentum tensor. The stationary metric reads

$$
\begin{aligned}
d s^{2}=- & e^{\frac{2 \nu}{c^{2}}}\left(d x^{0}\right)^{2}+r^{2} e^{\frac{2 \beta}{c^{2}}}\left(d \phi-\frac{\omega}{c^{3}}(r, z) d x^{0}\right)^{2} \\
& +e^{\frac{2 \alpha}{c^{2}}}\left(d r^{2}+d z^{2}\right)
\end{aligned}
$$

Here $x^{0}=c t$ is the rescaled time coordinate, and $r, z, \phi$ are cylindrical coordinates. We assume axial symmetry and employ the stress-momentum tensor

$$
T^{\alpha \beta}=\rho\left(c^{2}+h\right) u^{\alpha} u^{\beta}+p g^{\alpha \beta},
$$

where $\rho$ is the baryonic rest-mass density, $h$ is the specific enthalpy, and $p$ is the pressure. The 4 -velocity $u^{\alpha}$ is normalized, $g_{\alpha \beta} u^{\alpha} u^{\beta}=-1$. The coordinate (angular) velocity reads $\vec{v}=\Omega \partial_{\phi}$, where $\Omega=u^{\phi} / u^{t}$.

We assume a barotropic equation of state $p=p(\rho)$. To be more concrete, one can take the polytropic equation of state $p(\rho, S)=K(S) \rho^{\gamma}$, where $S$ is the specific entropy of fluid. Then one has $h(\rho, S)=K(S) \frac{\gamma}{\gamma-1} \rho^{\gamma-1}$. The entropy is assumed to be constant.

Define the square of the linear velocity

$$
V^{2}=r^{2}\left(\Omega-\frac{\omega}{c^{2}}\right)^{2} e^{2(\beta-\nu) / c^{2}} .
$$

The potentials $\alpha, \beta, \nu$, and $\omega$ satisfy equations that have been found by Komatsu, Eriguchi and Hachisu [3]. They constitute an overdetermined, but consistent, set of equations. The general-relativistic Euler equations are solvable, assuming an integrability condition - that the angular momentum per unit mass,

$$
j=u_{\phi} u^{t}=\frac{V^{2}}{\left(\Omega-\frac{\omega}{c^{2}}\right)\left(1-\frac{V^{2}}{c^{2}}\right)},
$$

depends only on the angular velocity $\Omega ; j \equiv j(\Omega)$. In such a case the Euler equations reduce to a generalrelativistic integro-algebraic Bernoulli equation, that embodies the hydrodynamic information carried by the continuity equations $\nabla_{\mu} T^{\mu \nu}=0$ and the baryonic mass conservation $\nabla_{\mu}\left(\rho u^{\mu}\right)=0$. It is given by the expression

$$
\ln \left(1+\frac{h}{c^{2}}\right)+\frac{\nu}{c^{2}}+\frac{1}{2} \ln \left(1-\frac{V^{2}}{c^{2}}\right)+\frac{1}{c^{2}} \int d \Omega j(\Omega)=C .
$$

\section{ROTATION LAWS}

The general-relativistic rotation law employed in the literature [3, 7] has the form

$$
j(\Omega)=A^{2}\left(\Omega_{c}-\Omega\right)
$$

where $A$ and $\Omega_{c}$ are parameters. In the Newtonian limit and large $A$ one arrives at the rigid rotation, $\Omega=\Omega_{c}$, 
while for small $A$ one gets the constant angular momentum per unit mass. A three-parameter expression for $j$ is proposed in [2].

Below we define a new family of rotation laws,

$$
j(\Omega) \equiv \frac{w^{1-\delta} \Omega^{\delta}}{1-\frac{\kappa}{c^{2}} w^{1-\delta} \Omega^{1+\delta}+\frac{\Psi}{c^{2}}},
$$

where $w, \delta, \kappa$ and $\Psi$ are parameters. The rotation curves $\Omega(r, z)$ ought to be recovered from the equation

$$
\frac{w^{1-\delta} \Omega^{\delta}}{1-\frac{\kappa}{c^{2}} w^{1-\delta} \Omega^{1+\delta}+\frac{\Psi}{c^{2}}}=\frac{V^{2}}{\left(\Omega-\frac{\omega}{c^{2}}\right)\left(1-\frac{V^{2}}{c^{2}}\right)},
$$

For $\delta \neq-1$, the general-relativistic Bernoulli equation (6) acquires a simple algebraic form

$$
\begin{gathered}
\left(1+\frac{h}{c^{2}}\right) e^{\nu / c^{2}} \sqrt{1-\frac{V^{2}}{c^{2}} \times} \\
\left(1-\frac{\kappa}{c^{2}} w^{1-\delta} \Omega^{1+\delta}+\frac{\Psi}{c^{2}}\right)^{\frac{-1}{(1+\delta) \kappa}}=C .
\end{gathered}
$$

We shall explain now the meaning and status of the four constants $w, \delta, \kappa$ and $\Psi$. Assume that there exists the Newtonian limit (the zeroth order of the post-Newtonian expansion - $0 \mathrm{PN}$ ) of the rotation law. This yields

$$
\Omega_{0}=\frac{w}{r^{\frac{2}{1-\delta}}} .
$$

Thus $w$ and $\delta$ can be obtained from the Newtonian limit. Moreover, the constant $w$ is any real number, while $\delta$ is nonpositive - due to the stability requirement [1] and satisfies the bounds $-\infty \leq \delta \leq 0$ and $\delta \neq-1$. These two constants can be given apriori within the given range of values. Let us remark at this point that the rotation law (8), and consequently the Newtonian rotation (11), applies primarily to single rotating toroids and toroids rotating around black holes. In the case of rotating stars one would have to construct a special differentially rotating law, with the aim to avoid singularity at the rotation axis.

The two limiting cases $\delta=0$ and $\delta=-\infty$ correspond to the constant angular momentum per unit mass $\left(\Omega_{0}=\right.$ $\left.w / r^{2}\right)$ and the rigid rotation $(\Omega=w)$, respectively. The Keplerian rotation is related to the choice of $\delta=-1 / 3$ and $w^{2}=G M$, where $M$ is a mass [9]. The case with $\delta=-1$ should be considered separately, but we expect that the reasoning will be similar.

The values of $\kappa$ and $\Psi$ are problematical. One possibility to get them is to apply the PN expansion. The rotation law in the $\mathrm{PN}$ expansion scheme should not be given apriori, but is expected to build up - in the subsequent orders of $c^{-2}$ - from the Newtonian rotation law. The Newtonian rotation curves are specified arbitrarily, but the next PN corrections should be defined uniquely. This is, however, a well known property of the post-Newtonian expansions, that they are non-unique. Damour, Jaranowski and Schäfer [8] demand that a test body rotating circularly in a Schwarzschild space-time satisfies exactly the Keplerian rotation law with $\Omega^{2}=G M / R^{3}$, where $R$ is the areal radius. Inspired by this we impose a Fixing Condition (F-Condition thereafter) - that a rotating infinitely thin disk made of dust in a Schwarzschild space-time satisfies exactly the Bernoulli equation and the Keplerian rotation law.

Consider a rotating, infinitely thin and weightless disk of dust in the Schwarzschild geometry. This is a textbook knowledge that there exists a stationary solution - each particle of dust can move along a circular trajectory of a radius $R$ with the angular velocity $\Omega=\sqrt{G M / R^{3}}$. We shall present this solution in conformal coordinates, using our formalism. The conformal Schwarzschild metric reads $d s^{2}=-\Phi^{2} / f^{2}\left(d x^{0}\right)^{2}+f^{4}\left(d r^{2}+d z^{2}+r^{2} d \phi^{2}\right)$, where $\Phi=1-G M /\left(2 c^{2} \sqrt{r^{2}+z^{2}}\right)$ and $f=1+$ $G M /\left(2 c^{2} \sqrt{r^{2}+z^{2}}\right)$. The angular velocity is equal to the Keplerian velocity $\Omega^{2}=G M /\left(\sqrt{r^{2}+z^{2}}{ }^{3} f^{6}\right)$ and $R=\sqrt{r^{2}+z^{2}} f^{2}$. The total energy per unit mass $\Psi$ vanishes for a test dust. Let the disk lie on the $z=0$ plane and assume the rotation law with $\delta=-1 / 3$ and $\kappa=3$ :

$$
\frac{w^{4 / 3} \Omega^{-1 / 3}}{1-\frac{3}{c^{2}} w^{4 / 3} \Omega^{2 / 3}}=\frac{V^{2}}{\Omega\left(1-\frac{V^{2}}{c^{2}}\right)} .
$$

Here $V^{2}=\Omega^{2} r^{2} f^{6} / \Phi^{2}$. Notice that $h=0$; the enthalpy per unit mass vanishes for dust. This is a simple exercise to show that $w=\sqrt{G M}$ and $\Omega^{2}=G M /\left(r^{3} f^{6}\right)$ solve both Eq. (12) and the Bernoulli equation (10); the constant in (10) equals to unity.

\section{1PN CORRECTIONS TO ANGULAR VELOCITY}

Taking into account the above, we shall prove that if $\kappa=(1-3 \delta) /(1+\delta)+\mathcal{O}\left(c^{-2}\right)$ and $\Psi=$ $4 c_{0}+\mathcal{O}\left(c^{-2}\right)$, where $c_{0}$ is the Newtonian hydrodynamic energy per unit mass, then the exact solution satisfies the first post-Newtonian (1PN) equations. We shall use the formalism of [3] and the rotation law (9), and recover most of the results obtained in the $1 \mathrm{PN}$ approach employed in [11]. Notice that if $\delta=-1 / 3$, then $\kappa=3$ - one recovers the coefficient in front of $w^{4 / 3} \Omega^{2 / 3}$ in (12) that is required by the $\mathrm{F}$ Condition.

The 1PN approximation corresponds to the choice of metric exponents $\alpha=\beta=-\nu=-U$ with $|U| \ll c^{2}[10]$. Define $\omega \equiv r^{-2} A_{\phi}$. The spatial part of the metric

$$
\begin{aligned}
d s^{2}=- & \left(1+\frac{2 U}{c^{2}}+\frac{2 U^{2}}{c^{4}}\right)\left(d x^{0}\right)^{2}-2 c^{-3} A_{\phi} d x^{0} d \phi \\
& +\left(1-\frac{2 U}{c^{2}}\right)\left(d r^{2}+d z^{2}+r^{2} d \phi^{2}\right) .
\end{aligned}
$$

is conformally flat. 
We split different quantities $\left(\rho, p, h, U\right.$, and $\left.v^{i}\right)$ into their Newtonian (denoted by subscript ' 0 ' and $1 \mathrm{PN}$ (denoted by subscript ' 1 ') parts. E.g., for $\rho, \Omega, \Psi$, and $U$ this splitting reads

$$
\begin{gathered}
\rho=\rho_{0}+c^{-2} \rho_{1}, \\
\Omega=\Omega_{0}+c^{-2} v_{1}^{\phi}, \\
\Psi=\Psi_{0}+\mathcal{O}\left(c^{-2}\right), \\
U=U_{0}+c^{-2} U_{1} .
\end{gathered}
$$

Notice that, up to the 1PN order,

$$
\frac{1}{\rho} \partial_{i} p=\partial_{i} h_{0}+c^{-2} \partial_{i} h_{1}+\mathcal{O}\left(c^{-4}\right),
$$

where the $1 \mathrm{PN}$ correction $h_{1}$ to the specific enthalpy can be written as $h_{1}=\frac{d h_{0}}{d \rho_{0}} \rho_{1}$. For the polytropic equation of state this gives $h_{1}=(\gamma-1) h_{0} \rho_{1} / \rho_{0}$.

Making use of the introduced above splitting of quantities into Newtonian $0 \mathrm{PN}$ and $1 \mathrm{PN}$ parts one can extract from Eq. (6) the 0PN- and 1PN-level Bernoulli equations. The $0 \mathrm{PN}$ equation reads

$$
h_{0}+U_{0}-\frac{\delta-1}{2(1+\delta)} \Omega^{2} r^{2}=c_{0},
$$

where $c_{0}$ is a constant that can be interpreted as the energy per unit mass. At the Newtonian level this is supplemented by the Poisson equation for the gravitational potential

$$
\Delta U_{0}=4 \pi G \rho_{0},
$$

where $\Delta$ denotes the flat Laplacian. The first correction $v_{1}^{\phi}$ to the angular velocity $\Omega$ is obtained from the perturbation expansion of the rotation law (9) up to terms of the order $c^{-2}$. Assuming that $\Psi_{0}=4 c_{0}$, one arrives at

$$
v_{1}^{\phi}=-\frac{2}{1-\delta} \Omega_{0}^{3} r^{2}+\frac{A_{\phi}}{r^{2}(1-\delta)}-\frac{4 \Omega_{0} h_{0}}{1-\delta},
$$

where we applied Eqs. (11) and (16).

Remember that in the Newtonian gauge imposed in the line element (13) the geometric distance to the rotation axis is given by $\tilde{r}=r\left(1-U_{0} / c^{2}\right)+\mathcal{O}\left(c^{-4}\right)$. It is enlightening to write down the full expression for the angular velocity, up to the terms $\mathcal{O}\left(c^{-4}\right)$ :

$$
\begin{aligned}
\Omega= & \Omega_{0}+\frac{v_{1}^{\phi}}{c^{2}}=\frac{w}{\tilde{r}^{2 /(1-\delta)}}-\frac{2}{c^{2}(1-\delta)} \Omega_{0}\left(U_{0}+\Omega_{0}^{2} r^{2}\right) \\
& +\frac{A_{\phi}}{r^{2} c^{2}(1-\delta)}-\frac{4}{c^{2}(1-\delta)} \Omega_{0} h_{0} .
\end{aligned}
$$

This expression reduces to

$$
\begin{aligned}
\Omega= & \Omega_{0}+\frac{v_{1}^{\phi}}{c^{2}}= \\
& \frac{w}{\tilde{r}^{2 /(1-\delta)}}-\frac{4}{r c^{2}(1-\delta)} \Omega_{0} h_{0},
\end{aligned}
$$

in the case of test fluids, at the symmetry plane $z=0$. For the dust, in the Schwarzschild geometry, we get

$$
\Omega=\Omega_{0}+\frac{v_{1}^{\phi}}{c^{2}}=\frac{w}{\tilde{r}^{3 / 2}}
$$

the $1 \mathrm{PN}$ correction to $\Omega_{0}$ is equal to $\frac{3 U_{0}}{2 c^{2}} \Omega_{0}$. Thus the FC condition is satisfied in the $1 \mathrm{PN}$ order.

After these consideration we are able to interpret the meaning of various contributions to the $1 \mathrm{PN}$ angular velocity $\Omega$. The first term is simply the Newtonian rotation law rewritten as a function of the geometric distance, as given at the $1 \mathrm{PN}$ level of approximation, from the rotation axis. The second term in (19) vanishes at the plane of symmetry, $z=0$, for circular Keplerian motion of test fluids in the monopole potential $-G M / R$. Thus it is sensitive both to the contribution of the disk self-gravity at the plane $z=0$ and the deviation from the strictly Keplerian motion. The third term is responsible for the geometric frame dragging. The last term represents the recently discovered dynamic anti-dragging effect; it agrees (for the monomial angular velocities $\Omega_{0}=r^{-2 /(1-\delta)} w$ ) - with the result obtained earlier in [11].

A comment on the term $-\frac{2}{c^{2}(1-\delta)} \Omega_{0}^{3} r^{2}$, that has been missing in [11]. The reason for this omission is following. There is a gauge freedom in choosing an integrability condition for the 1PN hydrodynamic equation; due to that the Bernoulli equation of the $1 \mathrm{PN}$ order is specified up to a function $F(r)$. We assumed in [11], in order to get the $1 \mathrm{PN}$ Bernoulli equation as in 10 , that $F(r)=0$; but that is not consistent with the F-Condition. It appears that the right value is $F(r)=-\Omega_{0}^{4} r^{4} /(1+\delta)$, which leads to the emergence of the term in question.

The vectorial component $A_{\phi}$ satisfies the following equation

$$
\Delta A_{\phi}-2 \frac{\partial_{r} A_{\phi}}{r}=-16 \pi G r^{2} \rho_{0} \Omega_{0}
$$

The 1PN Bernoulli equation does not influence the $1 \mathrm{PN}$ correction to the angular velocity. It has the form

$$
\begin{aligned}
c_{1}= & -h_{1}-U_{1}-\Omega_{0} A_{\phi}+2 r^{2}\left(\Omega_{0}\right)^{2} h_{0}-\frac{3}{2} h_{0}^{2} \\
& -4 h_{0} U_{0}-2 U_{0}^{2}-\frac{\delta-1}{4(1+\delta)} r^{4} \Omega_{0}^{4}+F(r),
\end{aligned}
$$

where $c_{1}$ is a constant. In order to derive (23) we again used $\Psi_{0}=4 c_{0}$. This result agrees with the $1 \mathrm{PN}$ calculation of [11] up to the term $F(r)$.

The $1 \mathrm{PN}$ potential correction $U_{1}$ can be obtained from

$$
\Delta U_{1}=4 \pi G\left(\rho_{1}+2 p_{0}+\rho_{0}\left(h_{0}-2 U_{0}+2 r^{2}\left(\Omega_{0}\right)^{2}\right)\right) .
$$

Equations (22) and (24) have been derived in 11] in the framework of $1 \mathrm{PN}$ approximation. They can be also obtained directly from the Einstein equations written for the metric (2), as derived e.g. in [3]. Here we recall a version similar to that used in [6]; it turns out to be more 
conveninent than the original form of [3]. The relevant equations read

$$
\begin{aligned}
\Delta \nu= & 4 \pi \frac{G}{c^{2}} e^{2 \alpha / c^{2}}\left[\rho\left(c^{2}+h\right) \frac{1+V^{2} / c^{2}}{1-V^{2} / c^{2}}+2 p\right] \\
& +\frac{1}{2 c^{4}} r^{2} e^{2(\beta-\nu) / c^{2}} \nabla \omega \cdot \nabla \omega-\frac{1}{c^{2}} \nabla(\beta+\nu) \cdot \nabla \nu
\end{aligned}
$$

and

$$
\begin{aligned}
\left(\Delta+\frac{2}{r} \partial_{r}\right) \omega= & -16 \pi \frac{G}{c^{2}} e^{2 \alpha / c^{2}} \rho\left(c^{2}+h\right) \frac{\Omega-\omega / c^{2}}{1-V^{2} / c^{2}} \\
& +\frac{1}{c^{2}} \nabla(\nu-3 \beta) \cdot \nabla \omega,
\end{aligned}
$$

where $\nabla$ denotes the "flat" gradient operator. The remaining Einstein equations yield corrections of higher orders.

In summary, we have shown that - for $-\infty<$ $w<\infty$ and $-\infty \leq \delta \leq 0, \delta \neq-1$ - the choice $\kappa=(3-\delta) /(1+\delta)+\mathcal{O}\left(c^{-2}\right)$ and $\Psi=4 c_{0}+\mathcal{O}\left(c^{-2}\right)$ in the rotation law (9) guarantees that if there exists an exact solution analytic in powers of $c^{-2}$, then it satisfies the $0 \mathrm{PN}$ and $1 \mathrm{PN}$ approximating equations.

One easily finds out that the rotation law (8) satisfies the generalized Rayleigh criterion [12] for stability $\frac{d j}{d \Omega}<$ 0 up to $1 \mathrm{PN}$ order, assuming that $\delta$ is strictly negative.

Comments on the $1 P N$ corrections to the angular velocity. In the following considerations we assume $w>0$, which means $\Omega_{0}>0$, but the reasoning is symmetric under the parity operation $w \rightarrow-w$. The specific enthalpy $h \geq 0$ is nonnegative, thence $-\frac{4 \Omega_{0} h_{0}}{1-\delta}$ is nonpositive the discovered in [11] instantaneous $1 \mathrm{PN}$ dynamic reaction slows the motion: it "anti-draggs" a system. In contrast to that, the well known geometric term with $A_{\phi}$ is positive [1], and the contribution $\frac{A_{\phi}}{r^{2}(1-\delta)}$ to the angular velocity is positive - it pushes a rotating fluid body forward. Thus the two terms in (18) counteract.

Dust is special - the specific enthalpy $h_{0}$ vanishes, hence dust test bodies are exposed only to the geometric effect - the frame dragging. Even more special is the rigid (uniform) rotation - the correction terms $v_{1}^{\phi}$ are proportional to $1 /(1-\delta)$ and they vanish, because now $\delta=-\infty$. Uniformly rotating disks are already known to minimize the total mass-energy for a given baryon number and total angular momentum [13]. The vanishing of the $1 \mathrm{PN}$ correction $v_{1}^{\phi}$ is their another distinguishing feature.

It follows from our discussion that assuming the FCondition, one has three free parameters: $w, \delta$ and $\Psi$; the parameter $\kappa$ is a given function of $\delta$. The full system of Einstein-Bernoulli equations can be solved numerically within this class of data and the resulting solutions are expected to possess $0 \mathrm{PN}$ and $1 \mathrm{PN}$ limits.

\section{CONCLUDING REMARKS}

We write down the general-relativistic rotation laws, recover the well known geometric dragging of frames and derive a full form of the two other weak-fields effects, including the dynamic anti-dragging effect of [11]. The latter can be robust according to the numerics of [11], but the ultimate conclusion requires a fully generalrelativistic treatment, that is the use of the new rotation laws. The frame dragging occurs - through the Bardeen-Petterson effect [16] — in some AGN's [17]. The two other effects can lead to its observable modifications in black hole systems with heavy disks.

In the weak field approximation of general relativity the angular velocity of toroids depends primarily on the distance from the rotation axis - as in the Newtonian hydrodynamics - but the weak fields contributions make the rotation curve dependent on the height above the symmetry plane of a toroid.

The new rotation laws would allow the investigation of self-gravitating fluid bodies in the regime of strong gravity for general-relativistic versions of Newtonian rotation curves. In particular, they can be used in order to describe stationary heavy disks in tight accretion systems with central black holes. These highly relativistic systems can be created in the merger of compact binaries consisting of pairs of black holes and neutron stars [14, 15], but they might exist in some active galactic nuclei.

The new general-relativistic rotation laws can be applied to the study of various open problems in the postNewtonian perturbation scheme of general-relativistic hydrodynamics. We demonstrate in this paper that an adaptation of the condition used in [8] ensures uniqueness up the $1 \mathrm{PN}$ order. Further applications include the investigation of convergence of the post-Newtonian pertubation scheme, as well as the existence of the Newtonian and post-Newtonian limits of solutions.

\section{ACKNOWLEDGMENTS}

PM acknowledges the support of the Polish Ministry of Science and Higher Education grant IP2012 000172 (Iuventus Plus). EM thanks Piotr Jaranowski for discussions on the PN approximations.
[1] J.-L. Tassoul, Theory of rotating stars Princeton, N. J.: Princeton University Press, 1978.

[2] F. Galeazzi, S. Yoshida, and Y. Eriguchi, Astronomy and Astrophysics 541, 156 (2012).
[3] H. Komatsu, Y. Eriguchi, and I. Hachisu, Mon. Not. R. Astron. Soc. 237, 355 (1989).

[4] J. M. Bardeen, Astrophys. J. 162, 71 (1970).

[5] E. Butterworth and I. Ipser, Astrophys. J. 200, L103 
(1969).

[6] S. Nishida and Y. Eriguchi, Astrophys. J. 427, 429 (1994).

[7] S. Nishida, Y. Eriguchi, and A. Lanza, Astrophys. J. 401 618 (1992).

[8] T. Damour, P. Jaranowski and G. Schäfer, Phys. Rev. 62, $044024(2000)$

[9] P. Mach, E. Malec and M. Piróg, Acta Phys. Pol. B44, 107 (2013).

[10] L. Blanchet, T. Damour, and G. Schäfer, Mon. Not. R. Astron. Soc. 242, 289 (1990).

[11] P. Jaranowski, P. Mach, E. Malec, and M. Piróg, Phys.
Rev. D91, 024039(2015)

[12] H. Komatsu, Y. Eriguchi, and I. Hachisu, Mon. Not. R. Astron. Soc. 239, 153 (1989).

[13] R. H. Boyer and R. W. Lindquist, Phys. Lett. 20, 504 (1966).

[14] F. Pannarale, A. Tonita and L. Rezzolla, Astrophys. J. 727, art. id. 95(2011).

[15] G. Lovelace, M. D. Duez, F. Foucart, L. E. Kidder, H. P. Pfeiffer, M. A. Scheel, B. Szilagyi, Class. Quantum Grav. 30, art. id. 135004 (2013).

[16] J. Bardeen and J. Petterson, Astrophys. J. 195, L65 (1975).

[17] J. Moran, ASP Conference Series 395, 87 (2008). 